\title{
CAPSULE COMMENTARIES \\ Capsule Commentary on Turner et al., The Impact of Hospitalist Discontinuity on Hospital Cost, Readmissions, and Patient Satisfaction
}

\author{
Kathlyn E. Fletcher, MD MA \\ Department of Internal Medicine, Clement J. Zablocki VAMC and Medical College of Wisconsin, Milwaukee, WI, USA.
}

J Gen Intern Med 29(7): 1055

DOI: $10.1007 / \mathrm{s} 11606-014-2836-7$

(c) Society of General Internal Medicine 2014

$I^{n}$ $\mathrm{n}$ this study, the authors evaluated the effect of hospitalist discontinuity on cost, readmission and patient satisfaction. ${ }^{1}$ Discontinuity was measured by modifying two tools that are typically applied in the outpatient rather than inpatient setting: the Usual Provider of Care Index $(\mathrm{UPC})^{2}$ and the Number of Physicians Index (NPI). ${ }^{3}$ The authors found that discontinuity was associated with increased costs, had no impact on patient satisfaction and trended toward fewer readmissions.

Given the frequent discussion about the potential impact of discontinuity, high quality studies are needed. This study provides an excellent template for such work. First, the authors used discontinuity measurements that can be generalized across settings and schedules. Second, they included important covariates such as night versus day admission and weekday versus weekend admission. Third, they used important outcomes: cost, readmission and patient experience. In addition, the authors adjusted their models for patient length of stay, which is essential in any work exploring discontinuity. Length of stay certainly influences discontinuity, and the reverse may also be true. Therefore, understanding and accounting for length of stay is imperative.

With respect to the curious finding that discontinuity may help prevent readmission, more work must be done. A prior multi-site study to explore the relationship between readmission and length of stay found that readmission rates were affected by both the individual patient length of stay and also by the hospital's average length of stay. ${ }^{4}$ Even though the authors of the present study adjusted for patient length of stay, they could not adjust for the hospital's average since it was a single site study. Therefore, the impact of length of stay may not be entirely accounted for in this study. On the other hand, the authors propose that the second look associated with discontinuity may be beneficial for patient care. ${ }^{5}$ Both of these theories should be evaluated in future work.

In conclusion, this study is a solid first step in exploring how discontinuity impacts patient care. It also offers reassurance to those who worry that the discontinuity inherent in hospitalist services is detrimental to care.

Conflict of Interest: The author has no conflicts of interest with this article.

Corresponding Author: Kathlyn E. Fletcher, MD MA; Department of Internal Medicine, Clement J. Zablocki VAMC and Medical College of Wisconsin, 8701 Watertown Plank Road, Milwaukee, WI 53226, USA (e-mail: kfletche@mcw.edu).

\section{REFERENCES}

1. Turner J, Hansen L, Hinami K, Christensen N, Peng J, Lee J, Williams MV, O'Leary KJ. The impact of hospitalist discontinuity on hospital cost, readmissions, and patient satisfaction. J Gen Intern Med. doi:10.1007/ s11606-013-2754-0.

2. Saultz Jw. Defining and measuring interpersonal continuity of care. Ann Family Med. 2003;1(3):134-43.

3. Steinwachs DM. Measuring provider continuity in ambulatory care: an assessment of alternative approaches. Med Care. 1979;17(6):551-65.

4. Kaboli PJ, Go JT, Hockenberry J, et al. Associations Between Reduced Hospital Length of Stay and 30-Day Readmission Rate and Mortality: 14Year Experience in 129 Veterans Affairs Hospitals. Ann Intern Med. 2012;157(12):837-45.

5. Wachter RM. Does continuity of care matter? No: discontinuity can improve patient care. West J Med. 2001;175(1):5. 